\title{
Optimum Flux-Detection in the Absence of a priori Knowledge about the Signal
}

\author{
Corrie Huijs ${ }^{1}$ and Jan J. Koenderink ${ }^{2}$ \\ 1 Department of Computer Science, Technical University Twente, Enschede, The Netherlands \\ 2 Department of Medical and Physiological Physics, Physics Laboratory, State University Utrecht, Utrecht, The Netherlands
}

\begin{abstract}
Detection systems based on photon counting have to discriminate between two types of fluctuations in the photon count: those resulting from statistical fluctuations (=noise) and those caused by changes in the radiance set by the source (=signal). In contrast with earlier studies on ways of discriminating noise from signal changes, no specific assumptions are made about the source. An optimal discrimination-method has been developed for a detector that has no prior information about the mean of the Poisson distribution that describes its input signal. Because the detector has no prior information at its disposal it has to assume an a priori probability for the mean in a unique and objective way and it has to estimate the actual mean using Bayes rule of inference. This new discrimination-method is discussed in the context of signal processing in the visual system, but is generally applicable in all systems where photon-noise is important.
\end{abstract}

\section{Introduction}

Photon counting is the basis of several detection procedures used in for example nuclear physics and in medical diagnostical methods such as scintigraphy. Signal processing in the visual system at low luminances also appears to be based on photon counting (Hecht et al., 1942). One of the problems in the above mentioned fields of application is how to eliminate the noise caused by statistical fluctuations of the radiation field. Often the methods used for eliminating noise are adhoc ones. We shall discuss and solve this problem in relation to vision, but the results are formulated in such a way that they can also be applied in other fields. Vision at low luminances appears to be based on the analysis of individual photon absorptions in the retinal receptors. A number of receptors cooperate in socalled retinal receptive fields that function rather like flux-detectors (Enroth-Cugell and Shapley, 1973). The initial stages of the visual system can be regarded as arrays of detectors covering the retinal surface, each detector having its own receptor-input-group or "aperture". The visual system has to abstract both structural and metrical information from the fluxes sampled by the apertures. Structural information concerns the spatial and temporal structure of the irradiance distribution, whereas metrical information concerns momentary local irradiance magnitudes. Individual detectors are assumed to process only metrical information. When the aperture is small the photon absorptions permit the extraction of large amounts of structural information but only little metrical information. In contradistinction a large aperture causes a coarse spatial resolution and reduces the structural information supplied by the array of detectors, whereas the available metrical information exceeds that for the smaller aperture. Therefore, in practice, we have to find a criterion which ensures that both structural and metrical information are at an optimum level (Snyder et al., 1977). We distinguish three types of fluxdetectors in this paper: change-detectors, incrementdetectors and decrement-detectors. All three discriminate statistical fluctuations in their inputs from variations in the parameters characterizing their input. The input parameters are determined by the set-up generating the input process and will be called intended fluxes. An optimum discrimination criterion will be derived for each type of detector. Optimum detection is defined relative to the maximum number of mistakes (false alarms) permitted.

Bouman and Ampt (1965), noting that the photon signal is a Poisson process the spread of which equals the square root of the average, proposed a square root scaling device as an optimum change-detector. These authors did not specify what they meant by optimality, 
Table 1

\begin{tabular}{|c|c|c|}
\hline & Detector & Experimenter \\
\hline $\begin{array}{l}\text { Prior } \\
\text { to } \\
\text { experiment }\end{array}$ & $\begin{array}{l}\text { * has no prior infor- } \\
\text { mation } \\
\text { * expects: } \\
\text { - potential flux } \\
\text { - potential sample }\end{array}$ & $\begin{array}{l}\text { * potentiality to control } \\
\text { intended flux }\end{array}$ \\
\hline $\begin{array}{l}\text { First } \\
\text { period } \\
\text { of } \\
\text { experiment }\end{array}$ & $\begin{array}{l}\text { * receives a sample } \\
\text { (physical realisation } \\
\text { of intended flux) }\end{array}$ & $\begin{array}{l}\text { * sets intended flux } \\
\text { * has no control over } \\
\text { resulting actual } \\
\text { sample }\end{array}$ \\
\hline $\begin{array}{l}\text { Between } \\
\text { periods }\end{array}$ & $\begin{array}{l}\text { * plates expectation } \\
\text { - inferred flux } \\
\text { - expected sample }\end{array}$ & $\begin{array}{l}\text { * makes decision about } \\
\text { change or no change } \\
\text { of intended flux }\end{array}$ \\
\hline $\begin{array}{l}\text { Second } \\
\text { period } \\
\text { of experi- } \\
\text { ment }\end{array}$ & $\begin{array}{l}\text { * receives new (actual) } \\
\text { sample (physical } \\
\text { realisation of new } \\
\text { intended flux) }\end{array}$ & * sets new intended flux \\
\hline $\begin{array}{l}\text { After } \\
\text { second } \\
\text { period }\end{array}$ & $\begin{array}{l}\text { * difference between } \\
\text { expected sample } \\
\text { and actual sample } \\
\text { determines output } \\
\text { status }\end{array}$ & $\begin{array}{l}\text { * reads output status of } \\
\text { detector } \\
\text { * evaluates output status } \\
\text { in terms of prior mani- } \\
\text { pulation of intended } \\
\text { flux: } \\
\text { Result: hit, miss, false } \\
\text { alarm or correct } \\
\text { rejection }\end{array}$ \\
\hline
\end{tabular}

neither did they prove the optimality of their squareroot-scaling device. Our analysis reveals that the best possible change-detector is rather similar to their square-root-scaler.

The use of optimum change-detectors results in different thresholds for increments and decrements. This difference depends upon the detection accuracy ("false alarm rate") chosen. In their psychophysical experiments Patel and Jones (1968) found differences in incremental and decremental thresholds which correspond with our theoretical results (see discussion). According to Cohn (1974) these differences are due to the fallacious assumption that the detector knows the "intended-flux", whereas in reality only the experimenter can have this knowledge. Our results are based upon the fact that a detector has to make its decision solely on the basis of actually sampled fluxes.

\section{Statement of the Problem}

\subsection{Assumptions and Definitions}

The detector receives an external signal, which can change at any moment, e.g. as a consequence of changes in local irradiance or eye movements. It is the task of the detector to signal such changes. Schematically, we have the following situation: An experimenter adjusts the generator of the signal (e.g. the intensity of an incandescent lamp can be set by regulating the electrical current through the filament) and notes the output signal of a detector. The detector samples the signal by counting the number of input events accumulated in concatenated periods of equal and fixed duration, and compares the succeeding samples. The sampling period is a parameter that characterizes the detector. The output signal (or "state") of the detector is some function of the sequential samples.

The experimenter determines the intended-flux, the value of which is not available to the detector. The samples can be seen as a realisation of the intendedflux. Table 1 shows the different situations during the experiment.

Because the detector may assume no structure a priori in the sequence of photon absorptions the detector can at best assume a uniform probability of occurence of a photon absorption: any prior expectation of a specific form that differs from a constant cannot be based on objective considerations. The photon input is assumed to be Poissonian, that is "completely random". This means that the probability, $P_{\text {pois }}(N \mid \lambda)$, that the number of input events in a fixed period will equal $N$ where $\lambda$ is the intended flux is given by

$P_{\text {pois }}(N \mid \lambda)=\frac{\lambda^{N} \cdot e^{-\lambda}}{N !}$.

As said before, the detector knows only the samples. A well-built detector can compute from samples which values of the intended-flux are the most likely ones. Such an estimate of the intended-flux will be called inferred-flux. Before any sample has been taken the detector can only expect a potential value of intendedflux which we shall call potential-flux.

The experimenter who controls the intended-flux also observes the state of the detector. The detector may assume two possible states, labelled: "change" and "no-change". An observer who is not informed about the intended-fluxes cannot evaluate those states, but the experimenter can split the detector decisions into four groups according to their correctness. Following the terminology of decision theory (van Trees, 1968; Green and Swets, 1966) we shall call the detector decision "change" a "hit" if it is correct (that means: conforms to the intended-fluxes) and a "false alarm" if it is incorrect. If the detector decides "nochange" this decision is called a "miss" if it is incorrect and a "correct rejection" if it is correct.

It is not possible to minimize the likelihood of the two types of wrong answer simultaneously, since they are interdependent. We define optimum performance as 
the one which minimizes the probability of a "miss" while keeping the false alarm probability below a given value. (The higher the false alarm probability the noisier the representation of the radiation pattern will be at the level of the detector array.) It will be appreciated that the optimum is reached when the false alarm rate equals the maximum value permitted. Therefore we talk about "the" false alarm rate (F.A.R.) instead of the maximum value of false alarm rate. These considerations lead to the formulation of the following three problems which will be solved in the sequel:

Problem I. To derive the optimum discrimination criterion, which (as indicated above) minimizes the probability of a "miss" for a given false alarm rate (F.A.R.), if the detector has no information at all about the "intended-flux" (optimum change-detector problem).

Problem IIA. To determine the optimum detection performance for a given F.A.R., if the detector knows only that the intended-flux either increases or stays constant. The values of possible changes are unknown (optimum increment-detector problem).

Problem IIB. As IIA but for decrements (optimum decrement-detector problem).

These problems will be solved with the F.A.R. as a parameter. The F.A.R. can be regarded as a confidence level.

\section{Mathematical Formulation}

3.1 The a priori Probability Density Function for the Potential-Flux

Jaynes (1968) has introduced a method based on "transformation groups" to arrive at objective prior probability distributions given certain kinds of a priori knowledge. In our case an objective a priori probability density function (p.d.f.) $P_{\mathrm{pr}}:(0, \infty) \rightarrow[0, \infty)$ can be obtained through the following reasoning:

Let $A$ and $B$ be two detectors observing the same Poisson process. As a spectator one notices that the two detectors employ different time-scales. Since the potential-flux is dependent on the time-scale chosen, the same process appears to have different parameters for the detector. Objectivity demands that the a priori p.d.f. should not depend on the fortuitous choice of time-scale. Let $P_{\mathrm{pr}}^{A}$ be the a priori p.d.f. assumed by the detector $A$ and $P_{\mathrm{pr}}^{B}$ the one assumed by the detector $B$. For every value of the potential flux, $\lambda_{p}$,

$P_{\mathrm{pr}}^{A}\left(\lambda_{p}\right)=P_{\mathrm{pr}}^{B}\left(\lambda_{p}\right)$.

This equation must hold because of the objectivity condition. The two different time-scales are connected with the different time units $t_{A}$ and $t_{B}$. Thus there is a constant $\alpha$ such that

$t_{A}=\alpha t_{B}$

If the potential-flux has a value $\lambda_{p}^{A}$ according to detector $A$ and a value $\lambda_{p}^{B}$ to detector $B$ then $\lambda_{p}^{A}=\alpha \lambda_{p}^{B}$. This leads us to conclude that the a priori probability that $\lambda_{p}^{A} \in[\lambda, \lambda+d \lambda]$ will be equal to the a priori probability that $\lambda_{p}^{B} \in[\alpha \lambda, \alpha \lambda+\alpha d \lambda]$ or:

$P_{\mathrm{pr}}^{A}(\lambda) d \lambda=P_{\mathrm{pr}}^{B}(\alpha \lambda) d \alpha \lambda$.

Combination of (2) and (4) gives the functional equation

$P_{\mathrm{pr}}^{A}(\lambda)=\alpha P_{\mathrm{pr}}^{A}(\alpha \lambda)$

with

$P_{\mathrm{pr}}(\lambda) \propto 1 / \lambda$

as its unique solution.

This hyperbolical p.d.f. is an "improper" one in the sense that it cannot be normalised. This presents no real problem, it is permissible to use this improper a priori p.d.f. in the derivation of a proper p.d.f. This will be done in Sect. 3.3.

\subsection{The a priori Probability for Sample Results}

From the a priori p.d.f. for the potential-flux one can derive which sample values are a priori the most likely. The likelihood is given by the relative probabilities:

$P_{\mathrm{pr}}^{*}(N) \propto 1 / N$.

$P$ is an improper probability function which can only be used to derive a proper a posteriori probability function (Sect. 3.4).

\subsection{The a posteriori Probability Density Function}

A sample yields a posteriori information about the intended-flux. The a posteriori probability density, $P_{\text {pos }}(\lambda \mid N)$, for the possible values of the intended-flux, can be derived by using Bayes rule of inference (Feller, 1970):

$P_{\text {pos }}(\lambda \mid N) d \lambda \cdot P_{\mathrm{pr}}^{*}(N)=P_{\mathrm{pois}}(N \mid \lambda) P_{\mathrm{pr}}(\lambda) d \lambda$.

From (1), (7), (8) one obtains:

$P_{\text {pos }}(\lambda \mid N) \propto\left[\lambda^{N-1} \cdot e^{-\lambda}\right] /[(N-1) !]$.

The proportionality constant can be found by normalisation; thus we obtain

$P_{\text {pos }}(\lambda \mid N)=\left[\lambda^{N-1} \cdot e^{-\lambda}\right] /[(N-1) !]=P_{\text {pois }}(N-1 \mid \lambda)$.

Consequently after a sample result " $N$ " the a posteriori probability density is a Poisson distribution with mean 


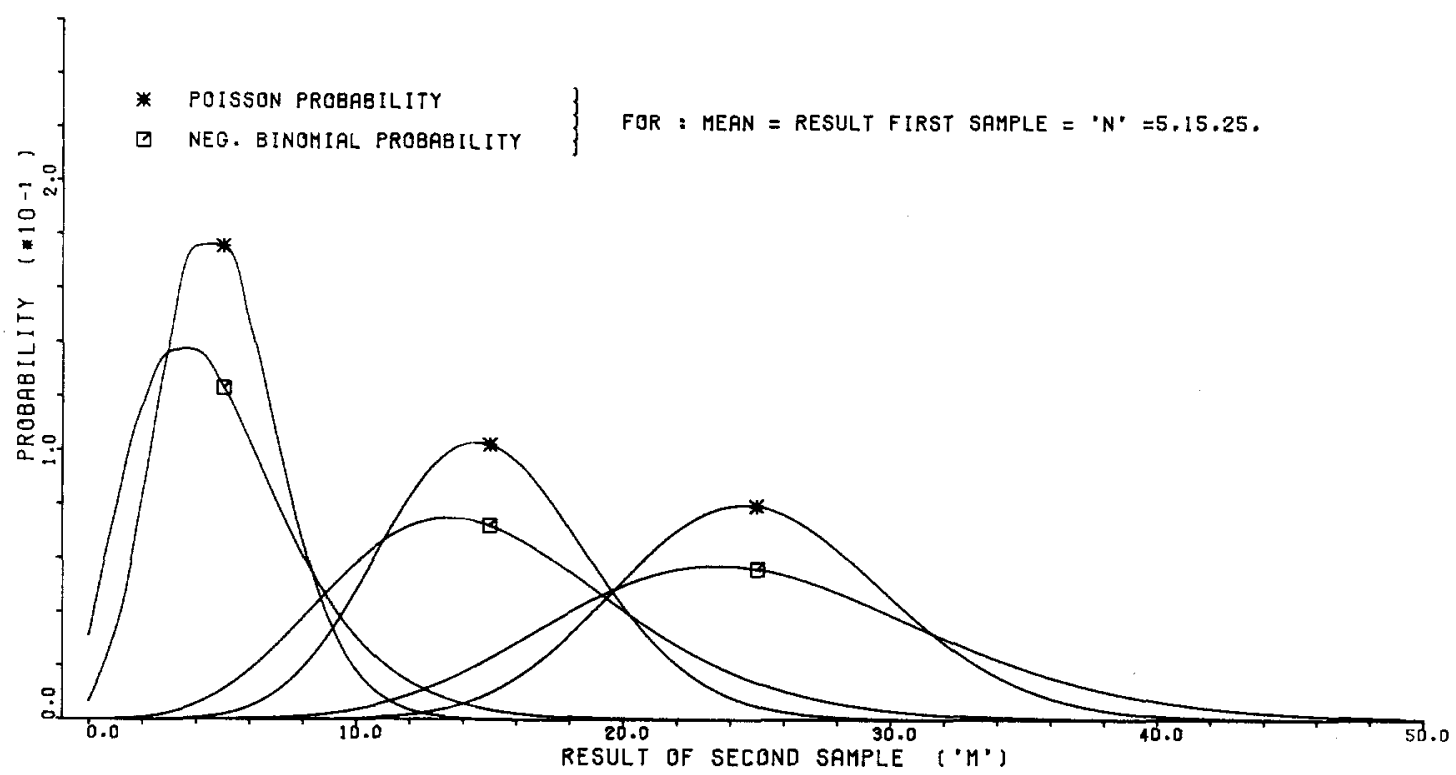

Fig. 1. Comparison of the poisson distribution and the negative binomial distribution

$N-1$ instead of $N$. The mean and variance of the $a$ posteriori p.d.f. are:

$\langle\lambda\rangle=\int_{0}^{\infty} \lambda \cdot \frac{\lambda^{N-1} \cdot e^{-\lambda}}{(N-1) !} d \lambda=N \int_{0}^{\infty} \frac{\lambda^{N}}{N !} e^{-\lambda} d \lambda=N$

and

$$
\begin{aligned}
\operatorname{var}= & \left\langle\lambda^{2}\right\rangle-\langle\lambda\rangle^{2}=N(N+1) \int_{0}^{\infty} \frac{\lambda^{N+1}}{(N+1) !} \\
& \cdot e^{-\lambda} d \lambda-N^{2}=N .
\end{aligned}
$$

The first of these results appears to be intuitively obvious, and in fact has often been assumed without being derived. Yet it depends crucially on the hyperbolic a priori density, a fact that is seldom appreciated.

\subsection{The a posteriori Probability Function for Sequential Samples}

The a posteriori probability function, $P_{\mathrm{pos}}^{*}(M \mid N)$, gives the probability that a sample result $N$ will be followed by the sample result $M$ :

$$
\begin{aligned}
P_{\mathrm{pos}}^{*}(M \mid N) & =\int_{0}^{\infty} P_{\mathrm{pois}}(M \mid \lambda) \cdot P_{\mathrm{pos}}(\lambda \mid N) d \lambda \\
& =\left(\begin{array}{c}
M+N-1 \\
M
\end{array}\right)(1 / 2)^{M+N} .
\end{aligned}
$$

The function $P_{\text {pos }}^{*}(M \mid N)$ is known as the Pascal distribution or negative binomial distribution. In Fig. 1 this distribution is shown for several values of $N$ together with the corresponding Poisson distribution. It can be seen that the variance of the Pascal distri- bution is larger than that of the Poisson distribution. The variance of the Pascal distribution is $2 N$ (that of the Poisson distribution $N$ ) and its mean is $N$ (Abramowitz, 1972). These values of mean and variance have an immediate intuitive meaning: After an input $N$ the inferred-flux has to be $N$ and the expected value of the actual flux therefore has to be $N$ also. Neither the parameter of the Poisson process (the intended-flux), nor the momentaneous value of this process (the actual sample) is known. The uncertainty in both of these quantities is $N$, so the total variance has to be $2 N$. The variance is twice the value that is often used on an intuitive basis, an immediate consequence of the hypothetical ignorance of the detector. Figure 1 also shows that the Pascal distribution is skew. The maximum value occurs at $M=N-1$ and $M=N-2$.

\subsection{The Discrimination Criterion}

After reception of a pair $N, M$ of contiguous and different samples the detector has to decide whether or not this difference has to be ascribed to a change in intended-flux. This means that for each $N$ there has to be a subset $n C(N)$ of $\mathbb{N}$ containing the values of the actual sample $M$ which must lead to the decision "no change". To keep the F.A.R. low $n C(N)$ has to contain the sample results which are the most likely to occur if the intended-flux has not changed. This means that $n C(N)$ has to be chosen in such a way that:

$\left.\begin{array}{l}K \in n C(N) \\ L \notin n C(N)\end{array}\right\} \Rightarrow P_{\text {pos }}^{*}(K \mid N) \geqq P_{\text {pos }}^{*}(L \mid N)$. 


\section{CHANGE-DETECTOR}

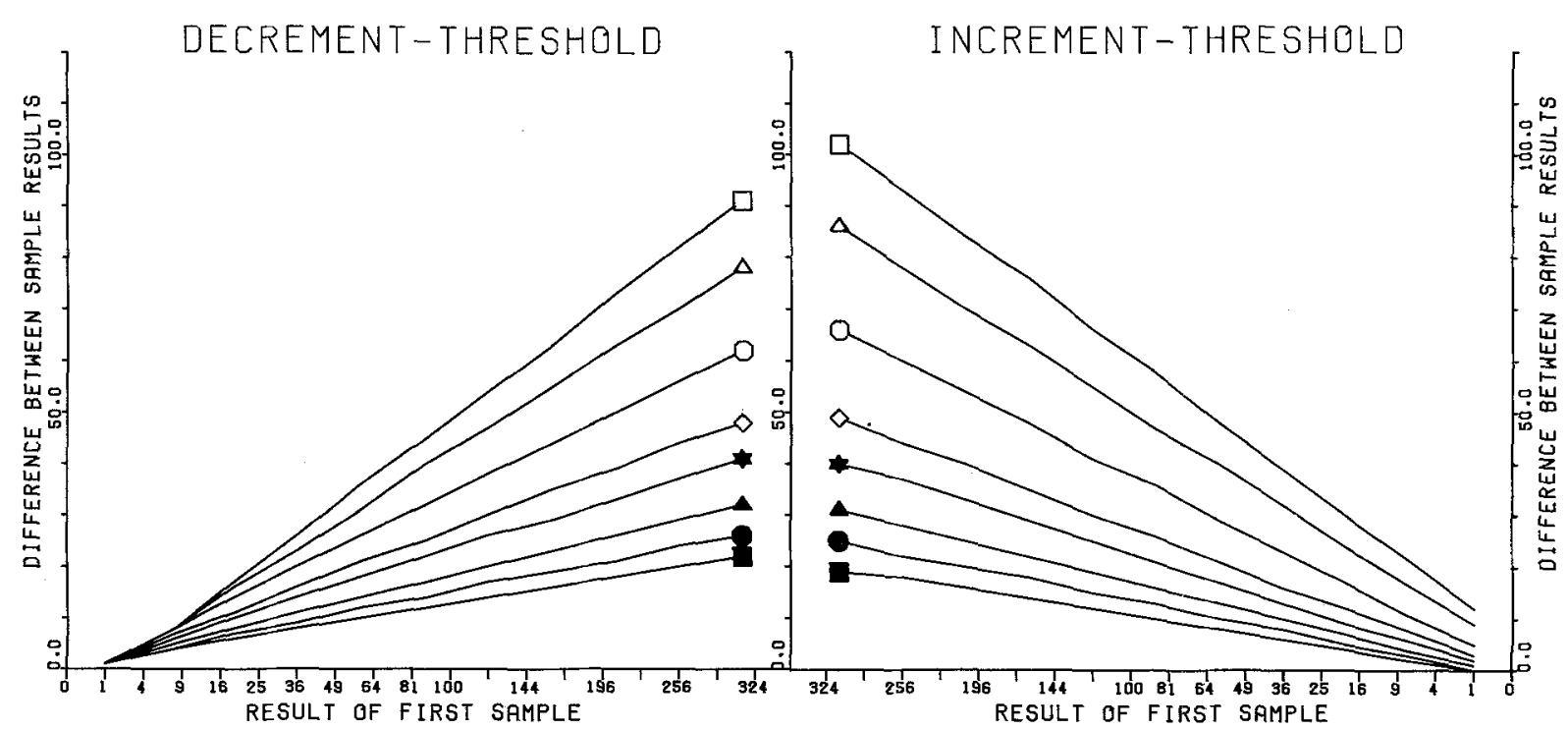

Fig. 2. Increment- and decrement-thresholds for a change-detector as a function of the value of the first sample with the F.A.R. as parameter. The different symbols stand for : $\boldsymbol{\nabla}$ threshold if false alarm rate $=0.4000 ; \bullet=0.3000 ; \Delta=0.2000 ; \star=0.1000 ; \diamond=0.0500 ; O=0.0100 ; \triangle=0.0010 ;$ $\square=0.0001$

Because of the monomodal shape of the Pascal distribution $n C(N)$ has to be an interval around $N$. The collection of subsets $n C(N)$ determines the discrimination criterion completely.

\subsection{Optimality Conditions}

The subsets $n C(N)$ can be used to calculate the probability that a false alarm will occur:

$P($ false alarm $)=\sum_{M \notin n C(N)} P_{\text {pos }}^{*}(M \mid N)$.

So the condition of limited false alarm is:

$$
\sum_{M \notin n C(N)} P_{\text {pos }}^{*}(M \mid N) \leqq \text { F.A.R. }
$$

The probability of a miss is minimized by choosing $n C(N)$ as small as possible for a fixed value of the false alarm rate. For a given F.A.R. the three given problems can be formulated mathematically as:

Problem I. Find for each $N$ the smallest $A_{I N}$ and $A_{D E}$ so that

$\sum_{M \notin n C(N)} P_{\mathrm{pos}}^{*}(M \mid N) \leqq$ F.A.R.

while

$n C(N)=\left[N-\Delta_{D E}, N+\Delta_{I N}\right]$.
Problem IIA. Find for each $N$ the smallest $A_{I N}$ so that:

$\sum_{M \notin n C(N)} P_{\text {pos }}^{*}(M \mid N) \leqq$ F.A.R.

while

$n C(N)=\left[0, N+\Delta_{I N}\right]$.

Problem IIB. Find for each $N$ the smallest $A_{D E}$ so that:

$\sum_{M \notin n C(N)} P_{\text {pos }}^{*}(M \mid N) \leqq$ F.A.R.

while

$n C(N)=\left[N-\Delta_{D E}, \infty\right)$.

\section{Derivation of the Optimal Criterion}

The method of Lagrange multipliers, which is usually used for this type of problem, cannot be used here because the probability of a miss, which is needed, is unknown. This is due to the fact that the detector knows nothing about the probability of changes in the intended-flux. For small values of $N$ and for given false alarm rate the interval of "no-change" $n C(N)$ can be found exactly by numerical computation, whereas for larger $N$ an analytic approximation can be used. Both methods are discussed. 


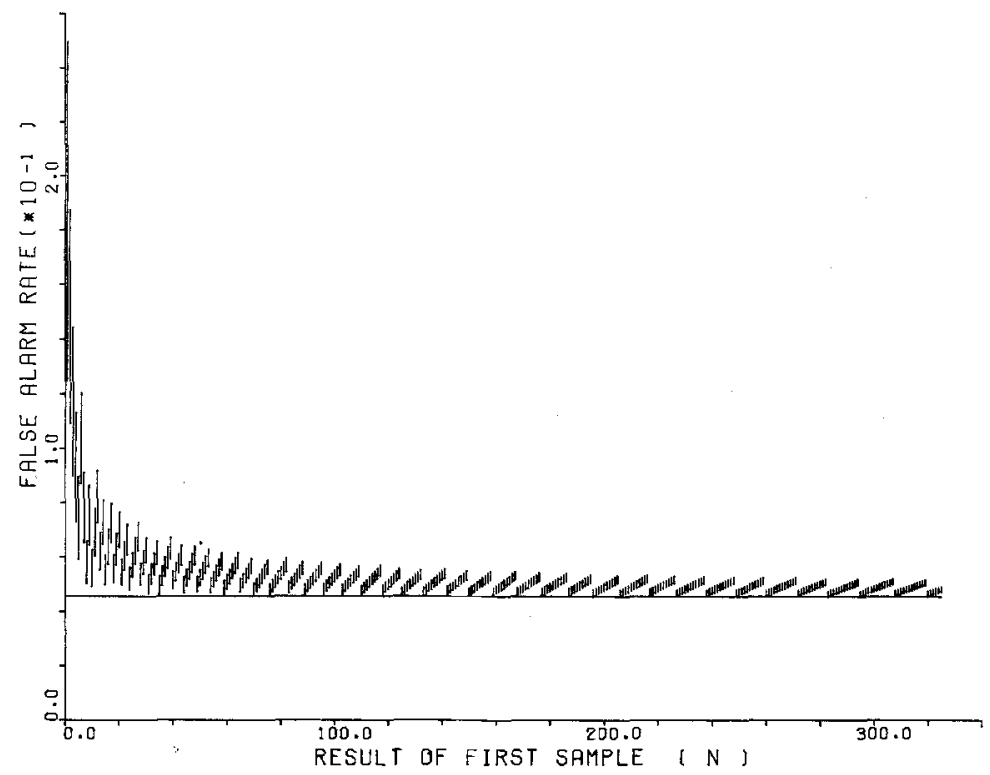

Fig. 3. F.A.R. intervals for which the incrementthreshold and the decrement-threshold of the change-detector are equal as a function of the value of the first sample

\subsection{The Numerical Solution}

Let F.A.R. $<1$ in the case of Problem I and F.A.R. $<0.5$ in the cases of the Problems IIA and IIB been given. If the value of F.A.R. is given the problems are defined completely. In the case of Problem I the numerical method starts for each $N$ with the interval that contains only $M=N-1$ [one of the maxima of $P_{\text {pos }}^{*}(\cdot \mid N) ; n C(N)$ is found by extending this interval until the sum of $P_{\text {pos }}^{*}$ over the interval exceeds $1-$ F.A.R.]. The extension is arranged in such a way that (13) holds.

Figure 2 shows the values of $\Delta_{I N}$ and $\Delta_{D E}$ by which the interval $n C(N)$ is determined. The differences between the values of $A_{I N}$ and $A_{D E}$ are dependent upon the F.A.R. and are due to the form of the Pascal distribution (Fig. 1). For each $N$ you have that $\Delta_{D E}$ is less than or equal $\Delta_{I N}$ if F.A.R. is small and $\Delta_{D E}$ is greater than or equal $\Delta_{I N}$ if F.A.R. is large. Therefore for each $N$ there has to be a value of the F.A.R. that leads to an equality of $\Delta_{D E}$ and $\Delta_{I N}$. Because of the discreteness of the Pascal distribution we find for each $N$ an interval of F.A.R. values which lead to equal increment- and decrement-thresholds. These intervals are shown in Fig. 3.

The numerical solution method is slightly different for the three problems. In Case IIA the procedure starts with an interval containing all elements less than or equal to $N$ and is extended to the right until the optimality condition (18) holds.

In the case of Problem IIB the procedure starts with an interval I, containing only 0 , which is extended to the right until

$\sum_{M \in I} P_{\mathrm{pos}}^{*}(M \mid N) \geqq 1-$ F.A.R. $n C(N)$ has to be the complement of the interval I before its last extension.

Figure 4 shows the values of $\Delta_{I N}$ and $\Delta_{D E}$ which determine the $n C(N)$ for the optimum incrementdetector and the optimum decrement detector, respectively. $A_{I N}$ and $\Delta_{D E}$ can be seen as thresholds which have to be exceeded before an increment or decrement is detected.

\subsection{Asymptotic Analytical Solution}

For large $N$ the Pascal distribution can be approximated by the Gaussian distribution with the same mean $(N)$ and variance $(2 N)$. It is well known that a false alarm rate criterion for a Gaussian distribution leads to a discrimination criterion proportional to the spread of the distribution, $2 N$. The proportionality constant which depends on the F.A.R. is plotted in Fig. 5 for the different cases.

\subsection{Comparison of Numerical and Analytical Results}

In Fig. 5 we see that in the case of the change-detector the approximate proportionality constants (upper line) are smaller than the proportionality constants calculated from Fig. 2 by the leasts-square-method. Because of the symmetry of the Gauss-distribution the approximated proportionality constants for the incrementthresholds equal those of the decrement-thresholds. The Camp-Paulson approximation (Johnson and Kotz, 1969) of the Negative Binomial distribution is asymmetrical. Therefore the Camp-Paulson approximation can, in contrast with the Gaussian approximation, give more insight in the differences between increment-thresholds and decrement-thresholds which 
DECREMENT-DETECTOR

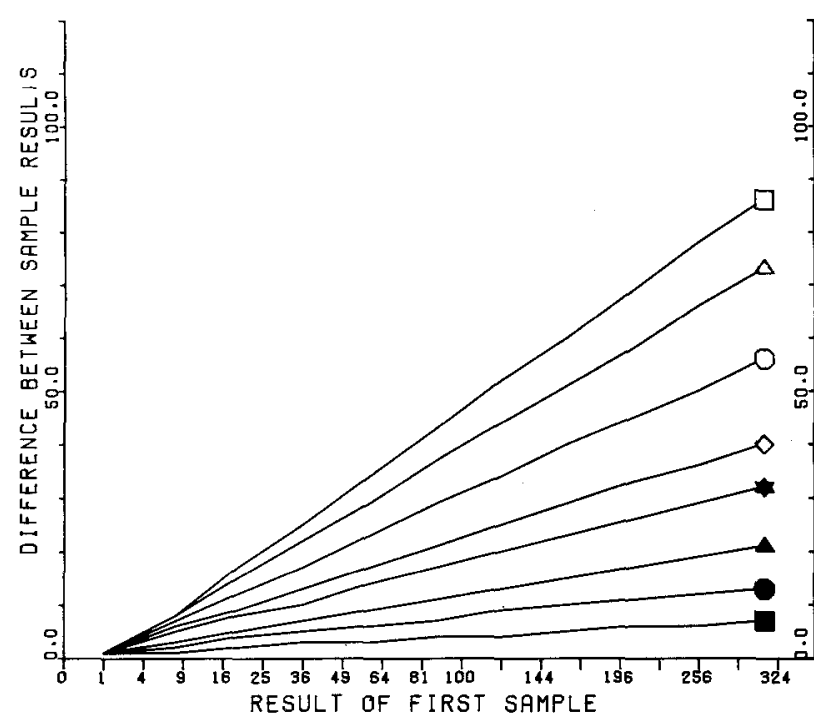

\section{NCREMENT-DETECTOR}

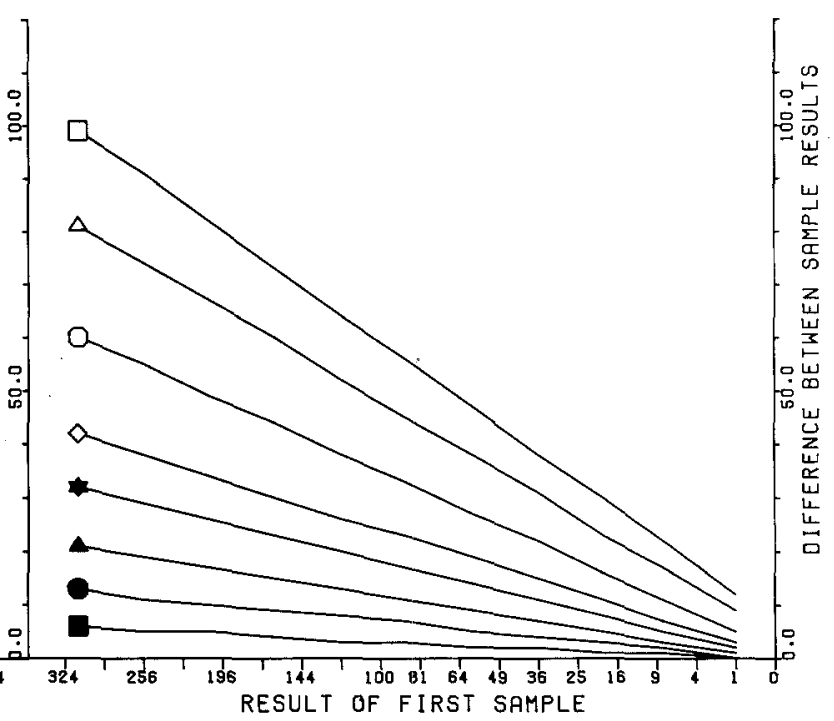

Fig. 4. Thresholds for an increment- and decrement-detector. The symbols have the same meaning as those in Fig. 2

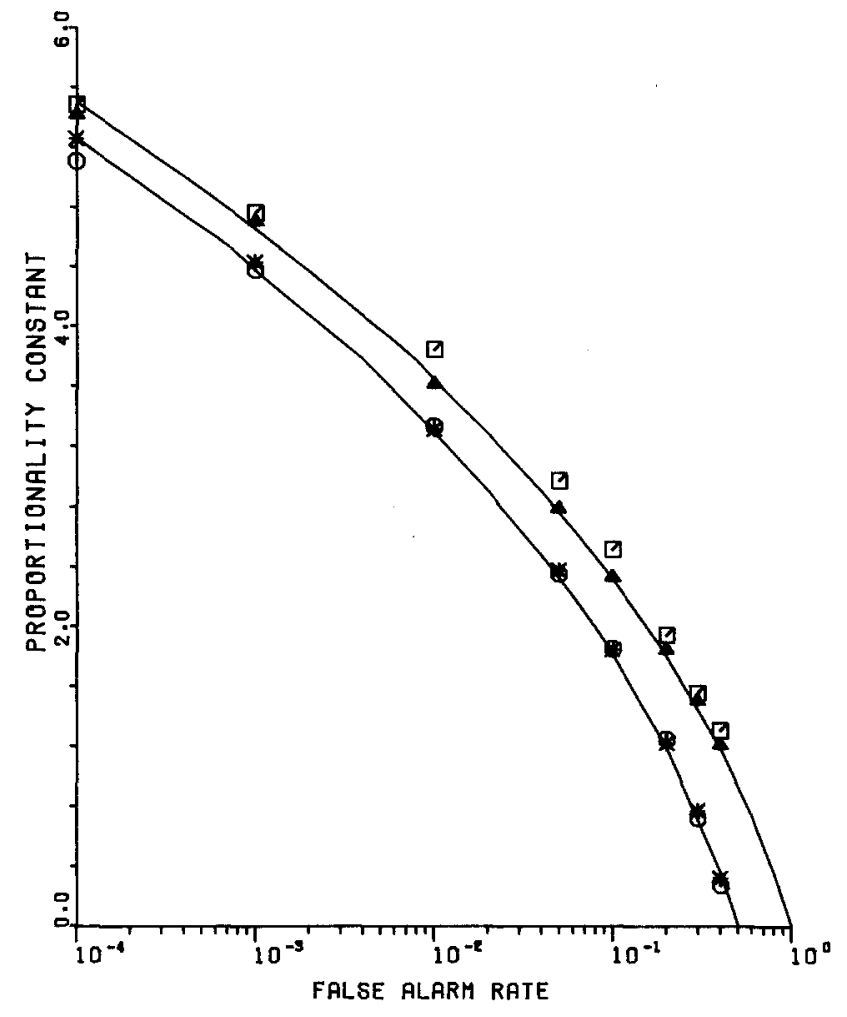

Fig. 5. The probability constant in the square-root relation between threshold and first sample result $(\square, \boldsymbol{\Delta}, *, O)$ least square approximations of this probability constant for respectively the increment threshold and decrement threshold of a change detector and the threshold of an increment detector and a decrement detector depend on the asymmetry of the Negative Binomial distribution. Qualitative understanding of the relation between the calculated and the approximated proportionality constants in Fig. 5 can be obtained by the use of Camp-Paulson approximation.

1) In the Camp-Paulson approximation given by Johnson and Kotz a minus symbol has to be replaced by a plus symbol in the formula for the integrationbound:

$$
P_{\text {pos } \downarrow}^{*}(M \leqq m \mid N)=(1 /(2 \pi)) \int_{-\infty}^{K} e^{-1 / 2 u^{2}} d u
$$

with

$$
\begin{aligned}
K= & \frac{1}{3}\left[\frac{(9 m+8)}{m+1}-\frac{(9 N-1)\{N /(m+1)\}^{1 / 3}}{N}\right] \\
& \cdot\left[\frac{\{N /(m+1)\}^{2 / 3}}{N}+\frac{1}{m+1}\right]-1 / 2
\end{aligned}
$$

Figure 2 shows that the increment-threshold is not exactly proportional to the square root of the first sample. The increment-threshold differs by an additive constant from a severe square-root relation. If we look at Fig. 4 we see that the same holds for the threshold of the increment-detector. The values of the translation constants are nearly the same in both cases. For the thresholds of the increment-detector and the decrement-detector the proportionality constants can well be calculated by the Gaussian approximation. 


\section{Discussion}

We have derived optimum discrimination criteria for the change-detector, the increment-detector and the decrement-detector. The principal features of the optimum detectors are the form of the discrimination criteria they have to use and the interrelation between increment thresholds and decrement thresholds. For a change-detector the discrimination criterion is given by the minimum differences between successive values of the actual-flux necessary to detect an increase or a decrease in the intended-flux. These two differences can be called increment and decrement threshold respectively. The values of these thresholds as well as their interrelation are dependent on the maximum permissible false alarm rate. For large values of the false alarm rate (F.A.R. $>0.06$ ) increment thresholds are smaller than decrement thresholds while for small values of the limit (F.A.R. $<0.045$ ) the situation is reversed. We could not obtain any simple analytic expression for the values of the exact thresholds, but as is shown in Figs. 2 and 4 the thresholds can be approximated rather well with the square root of " $N$ " multiplied by a constant. Thus we obtain the result that the square-root-scaler of Bouman and Ampt (1965) is not very different from the optimum changedetector for a false alarm rate of nearly $15 \%$.

It is of interest to know whether the performances of the optimum detectors is comparable to the performance of the real visual system. Psychophysical experiments in which the thresholds for incremental or decremental flashes are determined can in principle yield information about this. Yet the emperical results do not permit a conclusion because whereas many experiments have been done on increment thresholds, only a few have been done on decrement thresholds. Of greatest interest are the experiments in which both kinds of thresholds were determined under similar conditions (Patel and Jones, 1968; Short, 1966). These authors found that increment thresholds exceed the decrement thresholds. They used a small false alarm rate, F.A.R. $=0.025$, so their result corresponds to our prediction. We do not know of any experiments in which both thresholds are measured for different values of the false alarm rate.

From Fig. 5 you obtain that a F.A.R. of less than $1 \%$ forces you to use a criterion of about four times the square root of one sample to consider a change as "real". This criterion would change if you would change the experimental protocol. If the detector could sample the "background" for an extended time, it could infer the value of the intended flux with greater confidence and thus employ a less conservative criterion. In the limit of a very long exposure to the "background" and the task to detect a "flash" in a well marked interval, you would have the case of perfect prior knowledge of the intended flux of the background. Then the analysis invariably used by earlier authors would apply again. The case treated by us extends this simple paradigm (brief flash of well defined position in space and time upon an extended and enduring background) to the case of complete prior ignorance in which the intended flux could vary quite unpredictably from moment to moment.

Acknowledgement. This work was done at the Physical Laboratory of Utrecht University and was made possible through a grant from the Dutch Foundation for the Advancement of Pure Research (Z.W.O.).

\section{References}

Abramowitz, M., Stegun, I.A.: Handbook of mathematical functions, 9th edn. New York: Dover Publications 1972

Bouman, M.A., Ampt, C.G.F.: Fluctuation theory in vision and its mechanistic model. In: Excerpta Medica Int. Congress Series No. 125, Performance of the eye at low luminances. Proc. Symp. Delft 1965

Cohn, T.E.: A new hypothesis to explain why the increment exceeds the decrement threshold. Vision Res. 14, 1277-1279 (1974)

Enroth-Cugell, C., Shapley, R.M.: Flux, not retinal illumination is what cat retinal ganglion cells really care about. J. Physiol. 233, 311-326 (1973)

Green, D.M., Swets, J.A.: Signal theory and psychophysics. New York: Wiley 1966

Hecht, S., Shlaer, S., Pirenne, M. : Energy, quanta, and vision. J. Gen. Physiol. 25, 819-840 (1942)

Jaynes, E.T. : Prior probabilities. IEEE Trans. Syst. Sci. Cybern. SCC 4, 227-241 (1968)

Johnson, N.L., Kotz, S. Distributions in statistics: discrete distributions. Boston, Ma.: Hoghton Mifflin/Wiley 1969, pp. $122-142$

Patel, A.S., Jones, R.W.: Increment and decrement visual thresholds. J.O.S.A. 58, 696-699 (1968)

Short, A.D.: Decremental and incremental visual thresholds. I. Physiol. 185, 646-654 (1966)

Snyder, A.W., Laughlin, S.B., Stavenga, D.G.: Information capacity of eyes. Vision Res. 17, 1163-1175 (1977)

van Trees, H.L.: Detection, estimation, and modulation theory. New York: Wiley 1968

Received: April 11, 1983

Corrie Huijs

Jan J. Koenderink

Department of Medical and Physiological Physics

Physics Laboratory

State University Utrecht

Princetonplein 5

NL-3584 CC Utrecht

The Netherlands

Verantwortlich für den Textteil: Prof. Dr. W. Reichardt, Max-Planck-Institut für biologische Kybernetik, Spemannstr. 38 , D-7400 Tübingen. Verantwortlich für đen Anzeigenteil: E. Lückermann, G. Sternberg, Springer-Verlag, Kurfürstendamm 237, D-1000 Berlin 15, Fernsprecher: (030)882 1031, Telex: 01-85411. Springer-Verlag, Berlin. Heidelberg - New York- Tokyo.

Druck der Brühlschen Universitätsdruckerei, Gießen. Printed in Germany. - C Springer-Verlag GmbH \& Co KG Berlin Heidelberg 1983 